\title{
The transaction cost analysis and future development of the "blockchain + audit"industry- based on the analytic hierarchy model and the perspective of industry life cycle
}

\author{
Wenzhong $\operatorname{Tian}^{1 *}$ \\ ${ }^{1}$ Lanzhou University of Technology School of Economics and Management ,Lanzhou, Gansu, China
}

\begin{abstract}
The rise of "blockchain +" has a great impact on all fields and even the whole social and economic situation, and is no exception to the audit work. Blockchain has the characteristics of non-tampering, timestamp, grid consensus and so on, which is in line with the requirements of the audit work to pursue efficiency and effect. Under such a development background, the "blockchain+audit" industry arises from this. The integration of blockchain into audit engagements, in turn, changes the proportion of audit transaction costs. Based on this background, the Analytic Hierarchy Process (AHP) is used to analyze the transaction cost of audit. In view of the problems found in the current transaction cost analysis of "blockchain+audit", combined with the research on the future development trend based on the theory of technological innovation and industry life cycle, this paper puts forward some suggestions to effectively control the audit cost and improve the audit efficiency. The aim is to provide a practical reference basis for the better application of blockchain technology in auditing.
\end{abstract}

\section{INTRODUCTION}

Blockchain technology is widely known for its encrypted currency, but its block content is difficult to tamper with and can be permanently identified, which will have a wider application in the fields of authentication, supply chain management and financial transactions and auditing. Based on the theory of technological innovation and industrial life cycle,this paper attempts to study the industrial development trend of "blockchain+audit".

The new distributed bookkeeping technology represented by blockchain technology will promote the efficiency of transaction record and audit, and it is expected to fundamentally solve the credibility problem of the existing double-entry bookkeeping method. As a technological innovation, blockchain technology will promote the evolution of audit industry, and the focus of audit will be changed into system security, the correctness of system deployment and the attention of the use of technical specifications. Technical audit will become an important trend in the development of audit industry.

\section{Block chain and "Blockchain + Audit" Overview}

\subsection{Defective information function}

Blockchain is mainly constructed by computer network, connected through the Internet, and finally formed a network structure, that is ,"blockchain". It mainly includes data layer, network layer, consensus layer, incentive layer, contract layer and application layer. Its basic features include decentralization, independence and security. After the technology was first used in the field of finance, with the deepening of research, the tentacles of the technology have gradually extended to the field of accounting, especially to audit data and audit statements, etc. Then"blockchain+ audit"came into being.

\section{2"Blockchain + audit"}

Audit is an independent and responsible professional work. But the construction of "blockchain+audit"is the deficiency of adding block chain technology, optimizing and improving the traditional audit work in the traditional audit industry. "Blockchain+audit"is based on block books, based on text and numbers, information is stored in shared use. In this mode, information interaction is more smooth and data protection becomes more complete.

\section{Analysis of factors influencing audit transaction costs in the context of blockchain application}

\subsection{The impact of blockchain on audit}

\footnotetext{
*Wenzhong Tian :2298878966@qq.com
} 


\section{transaction costs}

The influencing factors of blockchain on audit transaction cost mainly include audit risk, audit process, audit subject, audit object and the construction and maintenance of blockchain platform.

\subsection{Transaction Cost Factor Analysis of "Blockchain + Audit" Based on Analytic Hierarchy Process}

In this paper, AHP is used to analyze the main factors affecting audit transaction costs in the context of blockchain application. According to the main steps of AHP evaluation, the following analysis is carried out.

\subsubsection{Build a hierarchical model}

Analytic hierarchy process (AHP) firstly sets up a hierarchy of decision-making problems according to their nature and subordination. Based on the above analysis, the following hierarchical model is constructed: 1.Layer audit risk: inherent risk, control risk, inspection risk; 2 . Audit process: preparation stage, implementation stage and termination stage;3.Audit subjects: Auditors master blockchain technology and audit software, professional ethics of auditors, and management level of the firm; 4. Audit object: company size, company internal control mechanism; 5. Blockchain platform construction and maintenance: Cooperate with technology enterprises and build blockchain team.

\subsubsection{Construct a paired comparison matrix}

The selected indicators are compared in pairs according to the degree of impact on audit transaction costs, which is usually expressed on a scale of 1-9, and a comparison matrix is constructed to make qualitative transformation into quantitative transformation. After inviting many enterprise project managers and accountants from accounting firms to score, a total of 16 valid questionnaires were received, and the judgment matrix was finally obtained.

\subsubsection{Hierarchical single sorting and consistency check}

1. Level 1 Indicators:Through calculation, the maximum eigenvalue is $\lambda \max =5.3514$, and the weight of each index is $\omega=(0.1384,0.0967,0.4702,0.0449$, 0.2498 ). The consistency index is $\mathrm{Ci}$, and the larger the $\mathrm{Ci}$, the more serious the inconsistency is. The random consistency index RI is introduced, and its standard value is shown in the table below:

Table1 Standard value of average random consistency

\begin{tabular}{|c|c|c|c|c|c|c|c|c|c|c|}
\hline $\mathrm{n}$ & 1 & 2 & 3 & 4 & 5 & 6 & 7 & 8 & 9 & 10 \\
\hline $\mathrm{RI}$ & 0 & 0 & 0.58 & 0.9 & 1.12 & 1.24 & 1.32 & 1.41 & 1.45 & 1.49 \\
& & & & & & & & & & \\
& & & & & & & & & & \\
\hline
\end{tabular}

The consistency ratio is calculated
$\mathrm{CR}=\frac{\mathrm{CI}}{\mathrm{RI}}=0.078<0.1$, Then we think the degree of consistency is acceptable.The single-level ranking of indicators is as follows: audit subject $=0.4702$, blockchain platform construction \& maintenance $=0.2498$, audit risk $=0.1384$, audit process $=0.0967$, audit object $=0.0449$.

2. Second level index:The maximum eigenvalue of $\mathrm{B} 1$ is $\lambda \max =3.0291$, The weight is $\omega_{1}=(0.0629,0.2654$, $0.6716)$, and the same goes for $\omega_{2}, \omega_{3}, \omega_{4}$, and $\omega_{5}$. Consistency test was conducted on B1, B2 and B3, and the calculated results $C R$ were all less than 1, indicating that the degree of consistency was within the acceptable range.

\subsubsection{Total hierarchical ordering}

In order to determine the importance of all factors to audit transaction costs, all index layers are sorted comprehensively according to their weights. Auditors' grasp of blockchain technology and audit software $=0.3435$, blockchain team construction $=0.2081$, inspection risk $=0.0930$, firm management level $=0.0886$, implementation stage $=0.0707$, cooperation with technical enterprises $=0.0416$, professional ethics of auditors $=0.0381, \quad$ Control risk $=0.0367$, company size $=0.0337$, termination stage $=0.0182$, company internal control mechanism $=0.0112$, inherent risk $=0.0087$, preparation stage $=0.0078$.

Thus, it can be seen that auditors' mastery of blockchain technology and audit software occupies the highest weight, followed by the indicators of blockchain team building. The influence degree of the three audit risks on audit transaction costs ranks lower, reflecting the changes in the weight of the influencing factors of audit transaction costs driven by blockchain.

\section{A Study on the Development Trend of "BlockChain+ Audit"Based on Industrial Life Cycle Theory}

From the industrial life cycle theory, Blockchain technology is essentially a driving force, In promoting the existing audit industry towards a new direction. According to industry life cycle theory, An industry is generally divided into four stages: input, growth, maturity and decline. In the early days, slow growth, growing faster, the growth rate of mature industry is the fastest, then it slows down. In general, technological innovation has always been regarded as the fundamental driving force of industrial evolution. After the production model based on the original technology has gradually entered the mature stage, technological innovation brings new ways of production, promote the upgrading of the original industry to the new industry as a whole. The growth of many industries is consistent with this law of production. Its production curve presents a $\mathrm{S}$ type as shown in figure1. The research of blockchain audit 
researchers coincides with the perspective of industrial evolution. On the one hand, researchers have fully recognized the huge advantages of blockchain technology in terms of data non-tampering and high levels of trust (Brown-liburd,Issa and Lombardi, 2015; Price Waterhouse Coopers, 2016) ${ }^{[1]-[2]}$. First, from the recording process itself, block chain technology records orderly and real-time records consistent with business logic - these independently accessible generated records are an important part of the audit manuscript, this will undoubtedly improve the efficiency of the audit. Second, in terms of the subsequent preservation and updating of records, once the transaction becomes a permanent part of the distributed accounts and is accepted by all participants in the blockchain network, will not be tampered with. This enables the audit itself to obtain a high credibility audit basis faster. Finally, high standardization of blockchain technology, make most of the data in the financial statements to achieve automatic verification. These features will ensure data security, a significant reduction in the time and cost (Deloitte, of the audit 2016) ${ }^{[3]}$. In addition to automating the audit process with blockchain technology, with the implementation of the block chain, the operational scope of the audit will be further expanded, the cost and efficiency of audit will be greatly improved - which will fundamentally improve the quality (Dai and Vasarhelyi,of audit 2016) ${ }^{[4]}$, reduce the possibility of artificial manipulation in existing books and audits. Blockchain technology reduces the cost of true and accurate identification of information, will undoubtedly promote the evolution of the audit industry.

Of course, the digital technology represented by blockchain has fundamentally impacted the business model of the current audit industry. Dai put forward the concept of audit phase 4.0 in 2016, and with the development of blockchain technology, the audit will move away from professional dependence, semi-automatic and even full automation has become the core feature of audit 4.0 stage. This also echoes to some extent the argument that blockchain technology will replace most of the existing audit functions. From the theory of industrial life cycle development, the mark of the second half of the mature period of the whole industry, as new production technologies emerge, the form of the production function of the original industry will change significantly, there will also be significant differences between the factors of production and the products produced. As blockchain technology becomes the foundation of books, the production process and object of audit industry will also change significantly. The focus of the audit changed from digital to technical systems for recording and generating numbers. For different types of block chains, there will be different audit strategies. In general, in terms of openness, blockchain is divided into public chain (Public blockchain) and private chain (Private blockchain) two kinds. Therefore, in the audit of the public chain, the audit focuses on whether the deployment of the public chain itself and its operating environment meet the requirements. Although the public chain is generally considered "completely decentralized", no person or institution can control or tamper with the reading and writing of the data, but in the event of manipulation, will have a negative impact on the entire system, and the general participants can not find the problems - which also puts forward higher requirements for the technical background of auditors. Compared to the public chain, private chain refers to a block chain open to individual individuals or entities, it has fewer nodes and only exposes its data in a limited network. The future, there will be more records of businesses and industries choosing to trade through private chains, private chain will also be the type of audit work in the future. Since the private chain is not completely decentralized, therefore, the audit of private chain has higher requirements. In addition to auditing the deployment and operating environments themselves, compliance of each transaction node during validation of data updates, will also become the focus of the audit. The audit process requires audit professionals to conduct compliance reviews of $\log$ content.

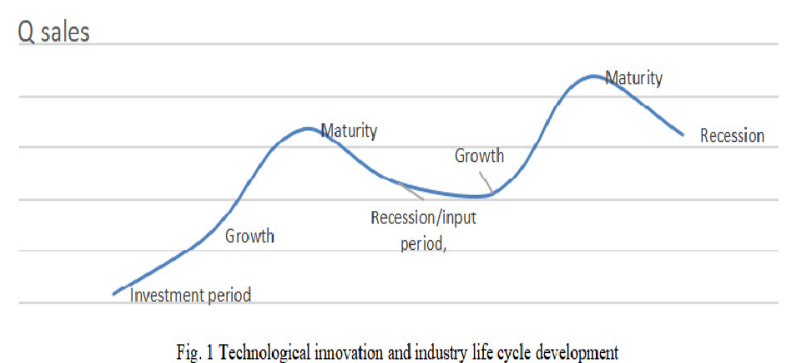

\section{Conclusion and Suggestions}

The audit industry should pay attention to system innovation and talent training in order to adapt to the evolution of the industry under the impact of technology. As technical audit becomes an important trend in the development of audit industry, the knowledge structure and career training direction of audit professionals will be very different from the past-and the talents with complex background of technology and finance will become an important factor restricting the development of the industry. At the project team level, based on the new audit objects and tasks, how to build a cross-background audit team, design audit team communication and quality control process, and clarify the positioning and division of labor between technicians and financial personnel will become an important problem in the future audit practice and research.

Under such changes, higher requirements have been put forward for audit subjects and accountants, and it has become a key factor to master audit software and be familiar with blockchain platform. Enterprises and accounting firms are recruiting high-level talents with the integration of audit and blockchain technology, and building their own blockchain team to maintain the blockchain platform has become the most important issue at present. The application of blockchain will reduce the cost of some elements, such as risk cost, etc. At the same time, it will also increase the cost of some elements, such 
as development costs. Enterprises and firms should form their own internal training mechanism, accelerate the talent training speed of the integration of audit and blockchain technology, reduce external hiring costs, and continue to output for themselves. This also reflects the change in the demand for talents. Therefore, to increase the social supply of such talents can greatly reduce the audit transaction cost.

The development and application of blockchain have a certain impact on audit risk, audit process, audit subject and object, etc. By constructing an indicator model to analyze the impact degree of each indicator on audit transaction cost, it is concluded that blockchain technology and related talents have become the key factors to reduce audit transaction cost. In general, the application of blockchain in audit can improve audit efficiency, reduce audit transaction costs, and have a profound impact on the audit industry. Blockchain technology is still evolving and will add even more value to various industries when it matures.

\section{References}

1. Brown-Liburd $\mathrm{H}$, Issa $\mathrm{H}$, Lombardi D.2015.Behavioral implications of Big Data's impact on audit judgement and decision making and future research directions $[\mathrm{J}]$. Accounting Horizons, 29(2):451-468

2. Bystrom H.2016. Blockchains. real-time accounting and the future of credit risk modeling[D].Sweden,Lund University,Department of Economics.

3. Deloitte L L P.2016.Blockchain Enigma.Paradox.Oppor-Tunity[R].London:The Creative Studio at Deloitte.

4. Dai J,Vasarhelyi M A.2016.Imagineering Audit 4.0[J].Journal of Emerging Technologies in Accounting,13(1):1-15 